Provided for non-commercial research and education use. Not for reproduction, distribution or commercial use.

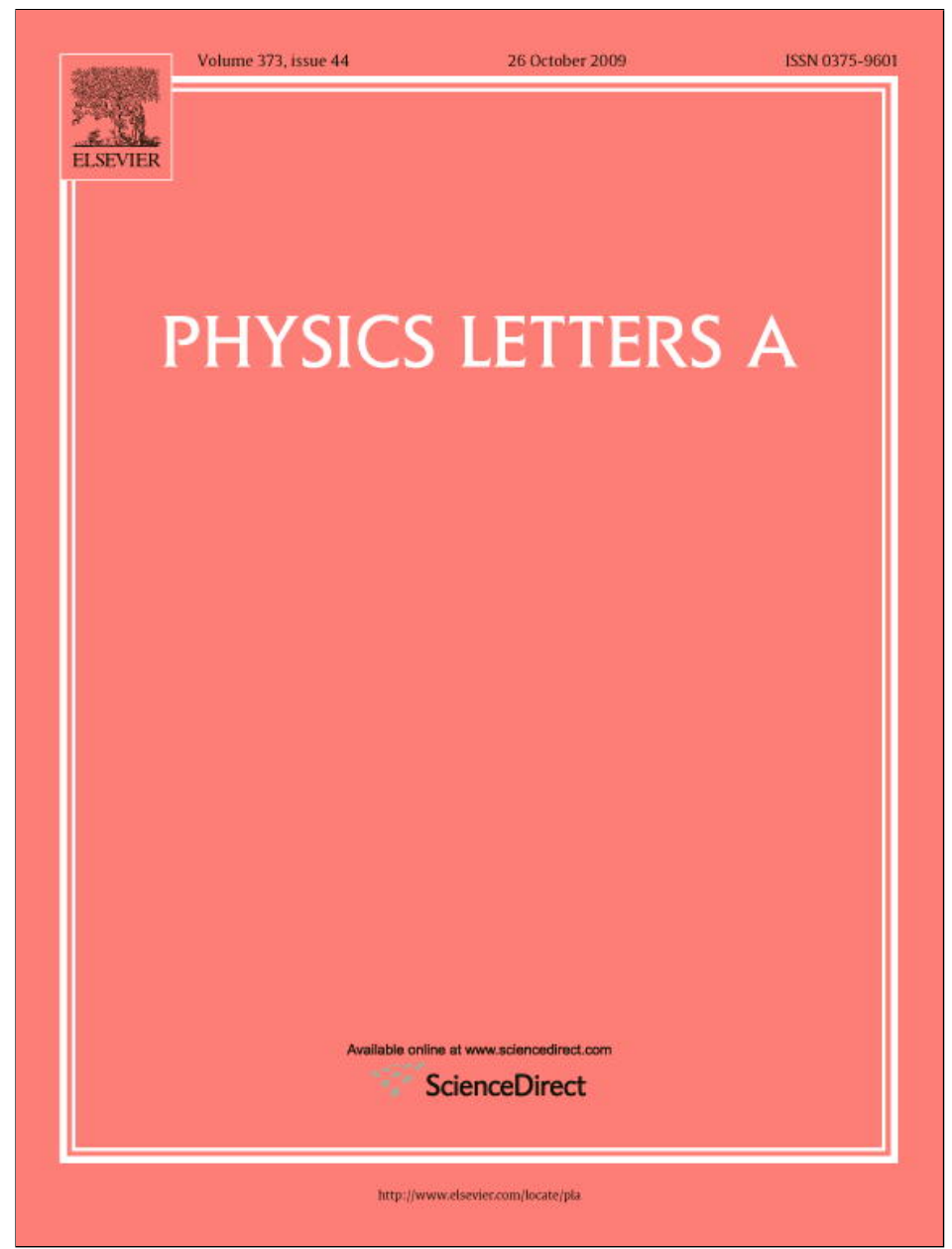

This article appeared in a journal published by Elsevier. The attached copy is furnished to the author for internal non-commercial research and education use, including for instruction at the authors institution and sharing with colleagues.

Other uses, including reproduction and distribution, or selling or licensing copies, or posting to personal, institutional or third party websites are prohibited.

In most cases authors are permitted to post their version of the article (e.g. in Word or Tex form) to their personal website or institutional repository. Authors requiring further information regarding Elsevier's archiving and manuscript policies are encouraged to visit:

http://www.elsevier.com/copyright 


\title{
Statistical theory of self-similarly distributed fields
}

\author{
Alexander Olemskoi ${ }^{\mathrm{a}, *}$, Irina Shuda ${ }^{\mathrm{b}}$ \\ a Institute of Applied Physics, National Academy of Sciences of Ukraine, 58, Petropavlovskaya St., 40030 Sumy, Ukraine \\ b Sumy State University, 2, Rimskii-Korsakov St., 40007 Sumy, Ukraine
}

\section{A R T I C L E I N F O}

\section{Article history:}

Received 10 July 2009

Accepted 19 August 2009

Available online 19 September 2009

Communicated by C.R. Doering

\section{PACS:}

02.20.Uw

05.30.Pr

12.40.Ee

Keywords:

Field theory

Generating functional

Jackson derivative

Deformation

\begin{abstract}
A B S T R A C T
A field theory is built for self-similar statistical systems with both generating functional being the Mellin transform of the Tsallis exponential and generator of the scale transformation that is reduced to the Jackson derivative. With such a choice, the role of a fluctuating order parameter is shown to play deformed logarithm of the amplitude of a hydrodynamic mode. Within the harmonic approach, deformed partition function and moments of the order parameter of lower powers are found. A set of equations for the generating functional is obtained to take into account constraints and symmetry of the statistical system.
\end{abstract}

(c) 2009 Elsevier B.V. All rights reserved.

\section{Introduction}

A formal basis of the statistical theory, using quantum field methods, is known to be a generating functional which presents the Fourier-Laplace transform of the partition function from the dependence on the fluctuating distribution of an order parameter to an auxiliary field [1]. Due to the exponential character of this transform, determination of correlators of the order parameter is provided by ordinary differentiation of the generating functional over auxiliary field.

Above scheme becomes inconsistent with passage from simple systems to complex ones because the phase space gets forbidden regions and the phase flow does not ensure statistical mixing [2]. As is known from the theory of critical phenomena, analytical description of complex systems is achieved in the presence of the scaling invariance only [3]. Because in this case the role of a basic function plays the power-law function instead of the exponential one, we need in use of the Mellin transform at constructing of the generating functional. Moreover, one should introduce the Jackson derivative as a generator of the scaling transformation instead of the ordinary derivation operator.

\footnotetext{
* Corresponding author

E-mail address: alex@ufn.ru (A. Olemskoi).
}

This Letter is devoted to building a field-theoretical scheme based on the use of both Mellin transform and Jackson derivative. The work is organized as follows. In Section 2 we adduce necessary information from the theory of quantum calculus. Section 3 is devoted to construction of the generating functional and finding its connection with related correlators. As the simplest example, the harmonic approach is studied in Section 4 to obtain the partition function and the order parameter moments of the first and second powers in dependence of the deformation parameter. In Section 5 we introduce pair of additive functional whose expansion into deformed series yields both Green functions and proper vertices. Moreover, we find here formal equations governing by the generating functional of systems possessing a symmetry with respect to a field variation and being subjected to an arbitrary constrain. Section 6 concludes our consideration.

\section{Preliminaries}

We begin by citing an information from the quantum calculus $[4,5]$ that will be needed below. A basis of this calculus is the dilatation operator $D_{x}^{\lambda}:=\lambda^{x \partial_{x}}$ being determined by the deformation parameter $\lambda$ and the differentiation operator $\partial_{x} \equiv \partial / \partial x$. Expanding formally the operator $D_{x}^{\lambda}$ into the Taylor series, it is easily to define its action onto the power-law function: $D_{x}^{\lambda} x^{n}=(\lambda x)^{n}$. Similarly, the expansion of an analytical function $f(x)$ shows that, in correspon- 
dence with the denomination, the operator $D_{x}^{\lambda}$ arrives at the dilatation $\lambda$ of the argument of this function: $D_{x}^{\lambda} f(x)=f(\lambda x)$. A set of eigen-functions of the dilatation operator is reduced to a homogeneous functions $h(x)$ defined by the equality $D_{\lambda} h(x)=\lambda^{q} h(x)$ with the self-similarity degree $q$. In general case, this function takes the form $h(x)=A_{\lambda}(x) x^{q}$ where a factor $A_{\lambda}(x)$ is obeyed the invariance condition $A_{\lambda}(\lambda x)=A_{\lambda}(x)$. It is convenient to pass from the dilatation operator to the Jackson derivative

$\mathcal{D}_{x}^{\lambda}:=\frac{D_{x}^{\lambda}-1}{(\lambda-1) x}$

in accordance with the commutation rule $\left[\mathcal{D}_{x}^{\lambda}, x\right]=D_{x}^{\lambda}$. The action the Jackson derivative onto the homogeneous function is given by the relations:

$\left(x \mathcal{D}_{x}^{\lambda}\right) h(x)=[q]_{\lambda} h(x), \quad[q]_{\lambda} \equiv \frac{\lambda^{q}-1}{\lambda-1}$.

Basic deformed number $[q]_{\lambda}$ represents a generalization of the exponent of the homogeneous function.

Principle peculiarity of self-similar statistical systems is that their consideration is based on the use of the deformed logarithm and exponential [2]

$\ln _{q}(x):=\frac{x^{1-q}-1}{1-q}, \quad \exp _{q}(x):=[1+(1-q) x]_{+}^{\frac{1}{1-q}}$,

where $[y]_{+} \equiv \max (0, y)$. Moreover, one needs to deform the sum and product as follows:

$x \oplus_{q} y=x+y+(1-q) x y$,

$x \otimes_{q} y=\left[x^{1-q}+y^{1-q}-1\right]_{+}^{\frac{1}{1-q}}$;

here, in the last equality $x, y>0$. Respectively, the deformed product of $n>1$ identical multipliers gives the expression of the deformed power-law function:

$\underbrace{x \otimes_{q} x \otimes_{q} \cdots \otimes_{q} x}_{n}=\left[n x^{1-q}-(n-1)\right]_{+}^{\frac{1}{1-q}}$.

Making use of the rules (4) shows that functions (3) are obeyed the conditions

$\ln _{q}\left(x \otimes_{q} x\right)=\ln _{q} x+\ln _{q} y, \quad \ln _{q}(x y)=\ln _{q} x \oplus_{q} \ln _{q} y ;$

$\exp _{q}(x+y)=\exp _{q}(x) \otimes_{q} \exp _{q}(y)$,

$\exp _{q}\left(x \oplus_{q} y\right)=\exp _{q}(x) \exp _{q}(y)$.

\section{Generating functional}

As mentioned above, the generating functional of self-similar systems is defined by the Mellin transform

$\mathcal{Z}_{q}\{J\}:=\int \mathcal{Z}_{q}\{\phi\} \phi^{J-1}\{\mathrm{~d} \phi\}$

$\phi^{J-1}\{\mathrm{~d} \phi\} \equiv \prod_{i=1}^{N} \phi_{i}^{J_{i}-1} \mathrm{~d} \phi_{i}$,

where index $i$ runs over lattice sites with number $N \rightarrow \infty .^{1}$ According to Ref. [2], the partition functional $\mathcal{Z}_{q}\{\phi\}=\exp _{q}(-S\{\phi\})$ is reduced to the deformed exponential with the exponent being

\footnotetext{
1 To escape complications related to continuum space [1] we use the lattice model.
}

inverse action $S=S\{\phi\}$ determined by the order parameter distribution. Functional $\mathcal{Z}_{q}\{J\}$ can be presented by the deformed series [5]

$$
\begin{aligned}
\mathcal{Z}_{\lambda}\{J\}= & \sum_{n=0}^{\infty} \frac{1}{[n]_{\lambda} !} \sum_{i_{1} \ldots i_{n}} \mathcal{Z}_{i_{1} \ldots i_{n}}^{(n)}\left(J_{i_{1}}-\lambda^{0}\right) \\
& \times\left(J_{i_{2}}-\lambda\right) \cdots\left(J_{i_{n}}-\lambda^{n-1}\right)
\end{aligned}
$$

where basic deformed factorial $[n]_{\lambda} !=[1]_{\lambda}[2]_{\lambda} \ldots[n]_{\lambda}$ is determined by the product of related numbers (2), while the coefficients

$\mathcal{Z}_{i_{1} \ldots i_{n}}^{(n)}=\left.\left(J_{i_{1}} \mathcal{D}_{J_{i_{1}}}^{\lambda}\right)\left(J_{i_{2}} \mathcal{D}_{J_{i_{2}}}^{\lambda}\right) \cdots\left(J_{i_{n}} \mathcal{D}_{J_{i_{n}}}^{\lambda}\right) \mathcal{Z}_{q}\{J\}\right|_{J_{i_{1}}, \ldots, J_{i_{n}}=1}$

are given by the Jackson derivative (1). By definition,

$\left.\left(J_{i} \mathcal{D}_{J_{i}}^{\lambda}\right) \phi^{J-1}\right|_{J_{i}=1}=\frac{\phi_{i}^{\lambda}-\phi_{i}}{(\lambda-1) \phi_{i}} \prod_{k \neq i} \phi_{k}^{J_{k}-1} \equiv \sigma\left(\phi_{i}\right) \prod_{k \neq i} \phi_{k}^{J_{k}-1}$,

where one denotes $\sigma\left(\phi_{i}\right) \equiv \ln _{2-\lambda}\left(\phi_{i}\right)$. Then, the Jackson derivation of the functional (7)

$$
\begin{aligned}
\left.\left(J_{i} \mathcal{D}_{J_{i}}^{\lambda}\right) \mathcal{Z}_{q}\{J\}\right|_{J_{i}=1} & =\int \mathcal{Z}_{q}\{\phi\} \sigma_{i}\left(\phi_{i}\right) \mathrm{d} \phi_{i} \prod_{k \neq i} \phi_{k}^{J_{k}-1} \mathrm{~d} \phi_{k} \\
& =\int \sigma_{i}\left(\phi_{i}\right) \mathcal{Z}_{q}\left(\phi_{i}\right) \mathrm{d} \phi_{i},
\end{aligned}
$$

arriving at the function $\mathcal{Z}_{q}\left(\phi_{i}\right):=\int \mathcal{Z}_{q}\{\phi\} \prod_{k \neq i} \phi_{k}^{J_{k}-1} \mathrm{~d} \phi_{k}$, yields the first moment of the deformed order parameter:

$\mathcal{S} \equiv\left\langle\sigma\left(\phi_{i}\right)\right|:=Z_{q}^{-1} \int \ln _{2-\lambda}\left(\phi_{i}\right) \mathcal{Z}_{q}\left(\phi_{i}\right) \mathrm{d} \phi_{i}$

$\left.Z_{q} \equiv \mathcal{Z}_{q}\{J\}\right|_{\{J\}=1}=\int \mathcal{Z}_{q}\{\phi\}\{\mathrm{d} \phi\}$

It represents the Tsallis entropy at condition that a field $\phi_{i}$ is reduced to the inverse probability to take a statistical state on a site $i$. Respectively, the kernel (9) determines the deformed correlator of an arbitrary order $n$

$$
\begin{aligned}
\left\langle\sigma\left(\phi_{1}\right) \ldots \sigma\left(\phi_{n}\right)\right\rangle & :=Z_{q}^{-1} \int \mathcal{Z}_{q}\left(\phi_{1}, \ldots, \phi_{n}\right) \prod_{m=1}^{n} \sigma\left(\phi_{i_{m}}\right) \mathrm{d} \phi_{i_{m}} \\
& =Z_{q}^{-1} \mathcal{Z}_{i_{1} \ldots i_{n}}^{(n)}
\end{aligned}
$$

where the function $\mathcal{Z}_{q}\left(\phi_{1}, \ldots, \phi_{n}\right):=\int \mathcal{Z}_{q}\{\phi\} \prod_{k \neq i_{1}, \ldots, i_{n}} \phi_{k}^{J_{k}-1} \mathrm{~d} \phi_{k}$ is used.

\section{Harmonic approach}

Within the standard field-theoretical scheme [1], the action $S=S_{0}+V$ is split into an unharmonic part $V=V\{\phi\}$ and the quadratic form $S_{0}:=\frac{1}{2 \Delta^{2}} \sum_{i} \phi_{i}^{2}$ determined by a variance $\Delta^{2}$. In accordance with Eq. (6), the partition functional is determined with the equality $\exp _{q}(-S)=\exp _{q}\left(-S_{0}\right) \otimes_{q} \exp _{q}(-V)$ where expansion over unharmonism with using the rule (10) gives the expression

$\mathcal{Z}_{q}\{J\}=\exp _{q}\left(-V\left\{J \mathcal{D}_{J}\right\}\right) \otimes_{q} \mathcal{Z}_{q}^{(0)}\{J\}$

of the generating functional

$$
\begin{aligned}
\mathcal{Z}_{q}\{J\} & :=\int \exp _{q}[-S\{\phi\}] \phi^{J-1}\{\mathrm{~d} \phi\} \\
& =\int \exp _{q}[-S\{\phi\}] \exp \{J \ln (\phi)\}\{\mathrm{d} \ln (\phi)\}
\end{aligned}
$$


in terms of the bare part $\mathcal{Z}_{q}^{(0)}\{J\}$ related to the action $S_{0}$. The principle difference of the equality (14) from corresponding expression for simple systems consists in deformation of both exponential operator of perturbation and its action onto the bare functional. Explicit expression of the generating functional (14) yields expansion into the deformed series (8) with the coefficients (9) determining the correlators (13).

Within framework of the harmonic approach, when the action $S_{0}=\frac{1}{2 \Delta^{2}} \sum_{i=1}^{N} \phi_{i}^{2}$ is reduced to the sum of the $N$ independent constituents, the functional $\mathcal{Z}_{q}^{(0)}\{J\}$ is expressed throughout the site functions $z_{q}^{(0)}\left(J_{i}\right)$ by means of the equality $\mathcal{Z}_{q}^{(0)}\{J\}=$ $z_{q}^{(0)}\left(J_{1}\right) \otimes_{q} z_{q}^{(0)}\left(J_{2}\right) \otimes_{q} \cdots \otimes_{q} z_{q}^{(0)}\left(J_{N}\right)$. Within the mean field approach, all multipliers coincide so that the use of Eq. (5) arrives at the expression of the generating functional (15) in terms of the site functions:

$\ln _{q}\left[\mathcal{Z}_{q}^{(0)}\{J\}\right] \simeq N \ln _{q}\left[z_{q}^{(0)}(J)\right]$.

As expected, the deformed logarithm of the partition functional proves to be additive value whose magnitude is determined by the cite constituent

$z_{q}^{(0)}(J)=\frac{1}{2}\left(\frac{2 \Delta^{2}}{1-q}\right)^{J / 2} \mathrm{~B}\left(Q, \frac{J}{2}\right), \quad Q \equiv \frac{2-q}{1-q}$.

Here, the B-function decays as $J^{-1}$ near the point $J=0$ transforming into the power-law dependence $J^{-Q}$ in the limit $J \rightarrow \infty$. As a result, the bare function (17) decays fast with the $J$ growing in vicinity of the point $J=0$ and then increases exponentially. At $J=1$, the dependence (17) gives the deformed partition function per one site

$z_{q}^{(0)}=\sqrt{\Delta^{2} / 2(1-q)} \mathrm{B}(Q, 1 / 2)$.

As a result, within the zero approach, the mean order parameter (12) takes the form

$\mathcal{S}^{(0)}=-(\lambda-1)^{-1}\left\{1-\left[\frac{\ln _{q}\left[z_{q}^{(0)}(\lambda)\right]}{\ln _{q}\left(z_{q}^{(0)}(1)\right)}\right]_{+}^{\frac{1}{1-q}}\right\}$.

In the information theory, the principle role is played by the Fisher matrix whose elements represent pair correlators of derivatives of the logarithm of the probability distribution function with respect to parameters of this function (in difference of the Tsallis entropy whose value gives a global measure of uncertainty, the Fisher matrix determines a local measure of information stored by the system) [6]. In the absence of a space correlations, such a measure is given by the moment of the second order $\mathcal{C} \equiv\left\langle\left[\sigma\left(\phi_{i}\right)\right]^{2}\right\rangle$ for which the use of Eqs. (13) and (9) arrives at the expression

$$
\begin{aligned}
\mathcal{C}^{(0)}= & (\lambda-1)^{-2}\left\{1-2\left[\frac{\ln _{q}\left[z_{q}^{(0)}(\lambda)\right]}{\ln _{q}\left(z_{q}^{(0)}(1)\right)}\right]_{+}^{\frac{1}{1-q}}\right. \\
& \left.+\left[\frac{\ln _{q}\left[z_{q}^{(0)}\left(\lambda^{2}\right)\right]}{\ln _{q}\left(z_{q}^{(0)}(1)\right)}\right]_{+}^{\frac{1}{1-q}}\right\} .
\end{aligned}
$$

At determination of the dependence of both moments (19) and (20) on the deformation $\lambda$, one needs to take into account that parameter $q$ is not free because a self-similarity condition restricts its value by the equation [7]

$$
\left(\lambda^{2-q}-1\right)\left(\lambda^{1-q}-1\right)=(\lambda-1)^{2} .
$$

As a result, variation of the deformation parameter $\lambda$ from 1 to $\infty$ arrives at growing the exponent $q$ since 0.382 to 0.5 . According to Figs. 1 and 2, hereby the specific partition function $z_{q}^{(0)} \sim 1$

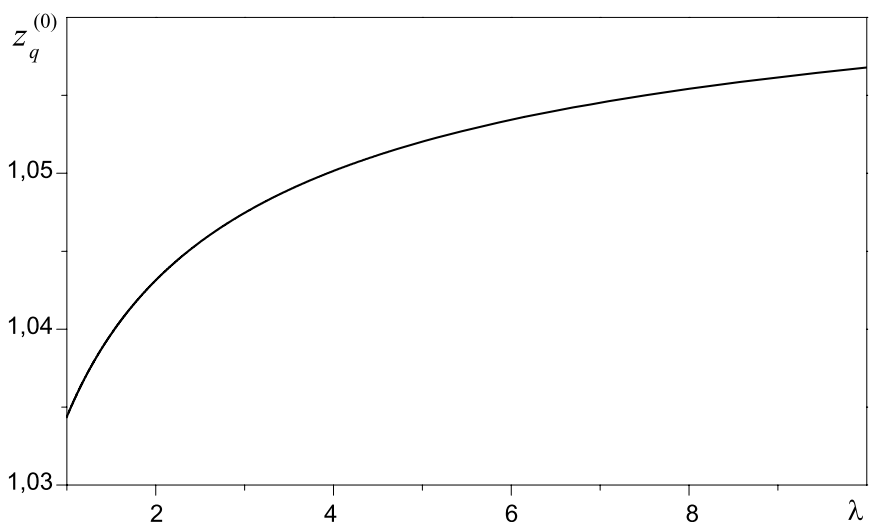

Fig. 1. Partition function (18) in dependence of the deformation parameter.

slightly increases, while the mean order parameter (19) keeps the zero value before the deformation parameter $\lambda=6.39$ and then increases monotonically. In contrast to this, the second order moment (20) displays slight maximum in the region $\lambda \geqslant 1$ that after downward excursion transforms into growing branch. From the point of view of the information theory [6], this means that before the value $\lambda=6.39$ a global measure of the information uncertainty $\mathcal{S}$ does not appears, while the local measure of information stored by the system $\mathcal{C}$ increases appreciably beginning since dilatations $\lambda \sim 3$.

What about the correlators of the higher orders, in thermodynamic limit $N \rightarrow \infty$, they are expressed in terms of the lower moments (19) and (20) with help of different uncouplings. As a result, the use of expression (14) allows one to build up a perturbation theory in analogy with the standard scheme [1].

\section{Field theory relations}

If a system consists of macroscopically independent parts 1 and 2 , then related actions are connected with the additivity condition $S_{1+2}=S_{1}+S_{2}$, whereas the deformed partition functional (15) whose kernel is determined by the expression $\exp _{q}\left(-S_{1+2}\right)=$ $\exp _{q}\left(-S_{1}\right) \otimes_{q} \exp _{q}\left(-S_{2}\right)$ is obeyed the deformed multiplicativity condition $\mathcal{Z}_{q}^{1+2}=\mathcal{Z}_{q}^{1} \otimes_{q} \mathcal{Z}_{q}^{2}$. Therefore, it is convenient to pass to the generating functional

$\mathcal{G}_{q}:=\ln _{q}\left(\mathcal{Z}_{q}\right)$

determined on the basis of the deformed logarithm that obeys the rules (6). As a result, the additivity condition $\mathcal{G}_{q}^{1+2}=\mathcal{G}_{q}^{1}+\mathcal{G}_{q}^{2}$ becomes to be satisfied so that the functional $\mathcal{G}_{q}=\mathcal{G}_{q}\{J\}$ can be understood as a thermodynamic potential. Because the latter depends on an auxiliary field $J$, one should use the Legendre transform

$\Gamma_{q}\{\phi\}:=\sum_{i} J_{i} \sigma_{i}-\mathcal{G}_{q}\{J\}, \quad \sigma_{i} \equiv \ln _{2-q}\left(\phi_{i}\right)$

to pass to a dependence on the order parameter $\phi$. This transform connects conjugated pair of thermodynamic potentials $\mathcal{G}_{q}\{J\}$ and $\Gamma_{q}\{\phi\}$ whose using arrives at the state equations

$\sigma_{i}=\mathcal{D}_{J_{i}}^{\lambda} \mathcal{G}_{q} \Leftrightarrow J_{i}=\mathcal{D}_{\sigma_{i}}^{\lambda} \Gamma_{q}$.

Similarly to the partition functional (7), above potentials are presented by the following deformed series:

$\mathcal{G}_{q}\{J\}=\sum_{n=1}^{\infty} \frac{1}{[n]_{\lambda} !} \sum_{i_{1} \ldots i_{n}} \mathcal{G}_{i_{1} \ldots i_{n}}^{(n)} J_{i_{1}} \ldots J_{i_{n}}$, 

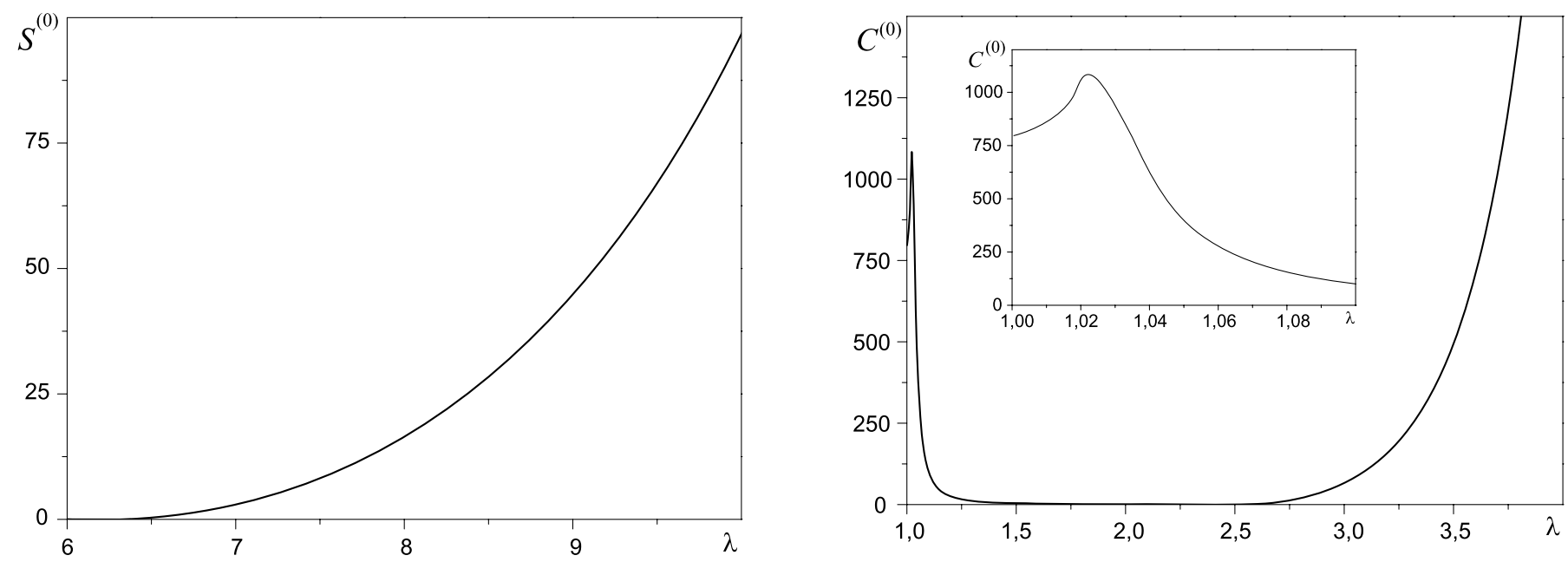

Fig. 2. Lower moments (19) and (20) of the deformed order parameter.

$$
\begin{aligned}
\Gamma_{q}\{\phi\} & =\sum_{n=1}^{\infty} \frac{1}{[n] \lambda !} \sum_{i_{1} \ldots i_{n}} \Gamma_{i_{1} \ldots i_{n}}^{(n)} \eta_{i_{1}} \cdots \eta_{i_{n}}, \\
\eta_{i_{m}} & \equiv \sigma_{i_{m}}-\lambda^{m-1} \mathcal{G}_{i_{m}}^{(1)}
\end{aligned}
$$

where in difference of the series (8) terms with index $n=0$ are absent (moreover, in Eq. (25) expansion is carried out over the field $J_{i_{m}}$ itself instead of the differences $J_{i_{m}}-\lambda^{m-1}$, while in Eq. (26) appears the difference $\left.\eta_{i_{m}} \equiv \sigma_{i_{m}}-\lambda^{m-1} \mathcal{G}_{i_{m}}^{(1)}, m=1, \ldots, n\right)$. With using the state equation (24), it is easily to convince that coefficients of the series (25) and (26) are connected with the same relations which take place in simple systems [1]. Within the diagrammatic representation, the kernels $\mathcal{G}_{i_{1} \ldots i_{n}}^{(n)}$ correspond to $n$-particle Green functions, while $\Gamma_{i_{1} \ldots i_{n}}^{(n)}$ determine proper vertices related [1].

Concluding this section, let us show that similarly to simple systems the generating functional (15) obeys a set of formal equations. The first of them is related to the system symmetry with respect to the field variation $\delta \ln \left(\phi_{i}\right)=\epsilon f_{i}\{\phi\}$ being proportional to an arbitrary functional $f_{i}\{\phi\}$ in the limit $\epsilon \rightarrow 0$. Due to this variation the integrand of the functional (15) obtains the multiplier $1+\epsilon\left[-\exp _{q}^{q}(-S) \partial S / \partial \ln \left(\phi_{i}\right)+J_{i}\right] f_{i}$, whereas the Jacobian of the passage from the variable $\ln \left(\phi_{i}\right)$ to $\ln \left(\phi_{i}\right)+\delta \ln \left(\phi_{i}\right)$ equals $1+\epsilon \partial f_{i} / \partial \ln \left(\phi_{i}\right)$ (the sum over repeated indices is meant). Collecting all multipliers before the factor $\epsilon$, in accordance with the invariance condition of the functional (15) one finds:

$$
\begin{gathered}
\left(f_{i}\left\{\frac{\delta}{\delta J}\right\}\left[\exp _{q}^{q}\left(-S\left\{\frac{\delta}{\delta J}\right\}\right) \frac{\partial S}{\partial \ln \left(\phi_{i}\right)}\left\{\frac{\delta}{\delta J}\right\}-J_{i}\right]\right. \\
\left.-\frac{\partial f_{i}}{\partial \ln \left(\phi_{i}\right)}\left\{\frac{\delta}{\delta J}\right\}\right) \mathcal{Z}_{q}\{J\}=0 .
\end{gathered}
$$

At $f_{i}\{\phi\}=$ const, this equation is simplified to take the form following immediately from the functional (15) after variation over the integration variable $\ln (\phi)$.

The second of the pointed equations allows one to take into account an arbitrary condition $F_{i}\{\ln (\phi)\}=0$ imposed on fields to be found. Accounting this condition is achieved by introducing the $\delta$-functional $\delta\{F\}$ into integrand of the last expression (15). As a result, the generating functional takes the elongated form

$$
\begin{aligned}
\mathcal{Z}_{q}^{(F)}\{J\}:= & \int \exp _{q}[-S\{\phi\}] \\
& \times \exp \{J \ln (\phi)+\lambda F\{\ln (\phi)\}\}\{\mathrm{d} \lambda\}\{\mathrm{d} \ln (\phi)\} .
\end{aligned}
$$

Variation of this expression over an auxiliary field $\lambda_{i}$ arrives at the desired equation

$F_{i}\left\{\frac{\delta}{\delta J}\right\} \mathcal{Z}_{q}^{(F)}\{J\}=0$

\section{Concluding remarks}

Following the standard scheme [1], we have considered the field theory of self-similar statistical systems whose states are distributed in accordance with the Tsallis exponential law. Because this distribution is characterized by the power-law tail, we have used the generating functional (7) based on the Mellin transform and the Jackson derivative (1) as the generator of the scaling transformation. Along this line, the role of the order parameter plays the mean value (12) of the deformed logarithm of the amplitude of a hydrodynamic mode. The use of the harmonic approach shows the specific partition function (18) slightly increases, the mean order parameter (19) keeps the zero value before the deformation $\lambda \geqslant 6$ and then increases monotonically, while the second order moment (20) displays slight maximum in the region $\lambda \geqslant 1$ and after downward excursion transforms into growing branch. Apart from the generating functional (7), we have introduced pair of the additive functional (22) and (23) whose expansions into deformed series (25) and (26) yield both Green functions and proper vertices. To take into account constraints and symmetry of the statistical system we have obtained Eqs. (27) and (29) for the generating functional.

The special peculiarity of our consideration is that the kernel of the Mellin transform (7) is reduced to the Tsallis exponential (3). But it is worthwhile to stress that the Tsallis deformation is not sole of possible procedures to obtain a distribution with power-law behavior. Another possibility is known to be given by the basic deformed distribution [8] that is invariant with respect to action of the Jackson derivative. ${ }^{2}$ Moreover, generalized three-parameter deformation procedure have been elaborated recently by Kaniadakis to obtain the whole set of power-law tailed distributions [9]. Building of generalized field theory based on above pointed distributions is in progress.

\section{References}

[1] J. Zinn-Justin, Quantum Field Theory and Critical Phenomena, Clarendon Press, Oxford, 1993.

\footnotetext{
2 In other words, eigen-value of the Jackson derivative operator defined on the basic deformed distribution equals 1 .
} 
[2] C. Tsallis, Introduction to Nonextensive Statistical Mechanics - Approaching a Complex World, Springer, New York, 2009.

[3] D. Sornette, Critical Phenomena in Natural Sciences, Springer, New York, 2001.

[4] C. Kassel, Quantum Groups, Springer, New York, 1995.

[5] V. Kac, P. Cheung, Quantum Calculus, Springer, New York, 2002.
[6] B.R. Frieden, Physics from Fisher Information: A Unification, Cambridge Univ. Press, Cambridge, 1998

[7] A.I. Olemskoi, A.S. Vaylenko, I.A. Shuda, Physica A 388 (2009) 1929.

[8] A. Lavagno, A.M. Scarfone, P. Narayana Swamy, J. Phys. A 40 (2007) 8635

[9] G. Kaniadakis, arXiv:0904.4180. 